\title{
$\varepsilon$-Aminocaproic acid (EACA) vs. tranexamic acid (TXA) in children undergoing complex cranial vault reconstruction for repair of craniosynostosis
}

\author{
Alexandra Borst ${ }^{1}$, Christopher Bonfield ${ }^{1}$, Poornachanda Deenadayalan ${ }^{2}$, Chi Le ${ }^{3}$, Meng \\ $\mathrm{Xu}^{3}$, Jenna Sobey ${ }^{1}$, and Srijaya Reddy ${ }^{1}$ \\ ${ }^{1}$ Monroe Carell Junior Children's Hospital at Vanderbilt \\ ${ }^{2}$ Vanderbilt University \\ ${ }^{3}$ Vanderbilt University School of Medicine
}

February 23, 2021

\begin{abstract}
INTRODUCTION: Children undergoing complex cranial vault reconstruction (CCVR) for craniosynostosis experience high rates of bleeding and transfusion, increasing risk for perioperative complications. $\varepsilon$-Aminocaproic acid (EACA) and tranexamic acid (TXA) are antifibrinolytic agents that have been shown to reduce intraoperative hemorrhage and transfusion requirements during CCVR. However, the relative efficacy of these two agents has not yet been evaluated. The aim of this study was to compare perioperative blood loss and transfusion rates in children receiving EACA vs. TXA. METHODS: All patients who underwent CCVR from September 2015 to December 2019 at a single center were retrospectively evaluated. Primary outcome measures included intraoperative estimated blood loss, postoperative drain output, transfusion volumes, and calculated blood loss. Secondary outcome measures included hematologic and coagulation parameters. RESULTS: 95 patients were included, with 47 patients in the EACA cohort and 48 patients in the TXA cohort. There were no significant differences in demographics, surgical outcomes, blood loss, transfusion requirement, or perioperative hematologic and coagulation laboratory values between the two cohorts. Adverse events were similar between the groups, but did include two seizure events and two thromboembolic events related to vascular access devices. DISCUSSION: We found no significant difference in blood loss, transfusion requirements, hematologic parameters, or outcomes between pediatric CCVR patients who received EACA vs. TXA. Further research is needed to define optimal antifibrinolytic dosing and duration of therapy. While standard laboratory parameters were similar between groups, future studies investigating coagulation-based and inflammatory assays may be useful in defining surgical-induced coagulopathy.
\end{abstract}

Title: $\varepsilon$-Aminocaproic acid (EACA) vs. tranexamic acid (TXA) in children undergoing complex cranial vault reconstruction for repair of craniosynostosis

\section{Authors:}

Alexandra J. Borst, MD

Division of Pediatric Hematology and Oncology Monroe Carell Jr. Children's Hospital at Vanderbilt

Christopher M. Bonfield, MD

Department of Neurological Surgery Monroe Carell Jr. Children's Hospital at Vanderbilt

1. Poornachanda S. Deenadayalan Vanderbilt University 
2. Chi H. Le, BS Vanderbilt School of Medicine

3. Meng $\mathrm{Xu}, \mathrm{MS}$

Department of Biostatistics Vanderbilt School of Medicine

Jenna H. Sobey, MD

Division of Pediatric Anesthesiology

Monroe Carell Jr. Children's Hospital at Vanderbilt

Srijaya K. Reddy, MD, MBA

Division of Pediatric Anesthesiology

Monroe Carell Jr. Children's Hospital at Vanderbilt

Corresponding Author:

Alexandra J. Borst, MD

Division of Pediatric Hematology and Oncology

Monroe Carell Jr. Children's Hospital at Vanderbilt

2200 Children's Way, $6^{\text {th }}$ Floor DOT

Nashville, TN 37232

(615) 936-1762 (phone), (615) 936-1767 (fax)

alexandra.borst@vumc.org

Manuscript Word Count: 2,521

Abstract Word Count: 246

Keywords: antifibrinolytic, craniosynostosis, bleeding, transfusion, coagulation

Running Title: Antifibrinolytics pediatric craniosynostosis repair

Tables and Figures: 6 Tables, 1 Supporting Information File

Abbreviations:

\begin{tabular}{ll}
\hline Abbreviation & Full Term \\
\hline ASA & American Society of Anesthesiologists physical status classification \\
CBL & calculated blood loss \\
CCVR & complex cranial vault reconstruction \\
EACA & c-aminocaproic acid \\
EBV & estimated blood volume, \\
ERCV & estimated red cell volume \\
FFP & fresh frozen plasma \\
FOA & frontal-orbital advancement \\
HCT & hematocrit \\
HER & electronic health record \\
HIPAA & Health Insurance Portability and Accountability Act \\
ICU & intensive care unit \\
INR & international normalized ratio \\
IQR & interquartile range \\
LOS & length of stay \\
MCJCHV & Monroe Carell Jr. Children's Hospital at Vanderbilt
\end{tabular}




\begin{tabular}{ll}
\hline Abbreviation & Full Term \\
\hline PT & prothrombin time \\
PTT & activated partial thromboplastin time \\
RBC & red blood cell \\
SD & standard deviation \\
TXA & tranexamic acid \\
\hline
\end{tabular}

\section{Meeting Abstract Publication:}

This research is being presented in poster abstract form as the following:

Borst, A.J., Bonfield, C.M., Deenadayalan, P.S., Le, C.H., Helmer Sobey, J., and Reddy, S.K. ع-aminocaproic acid (EACA) vs. tranexamic acid (TXA) in children undergoing complex cranial vault reconstruction for repair of craniosynostosis. Poster to be presented at: Society for Pediatric Anesthesia Meeting; 2021 Feb 27; virtual meeting platform.

\section{Abstract}

INTRODUCTION: Children undergoing complex cranial vault reconstruction (CCVR) for craniosynostosis experience high rates of bleeding and transfusion, increasing risk for perioperative complications. $\varepsilon$-Aminocaproic acid (EACA) and tranexamic acid (TXA) are antifibrinolytic agents that have been shown to reduce intraoperative hemorrhage and transfusion requirements during CCVR. However, the relative efficacy of these two agents has not yet been evaluated. The aim of this study was to compare perioperative blood loss and transfusion rates in children receiving EACA vs. TXA.

METHODS: All patients who underwent CCVR from September 2015 to December 2019 at a single center were retrospectively evaluated. Primary outcome measures included intraoperative estimated blood loss, postoperative drain output, transfusion volumes, and calculated blood loss. Secondary outcome measures included hematologic and coagulation parameters.

RESULTS: 95 patients were included, with 47 patients in the EACA cohort and 48 patients in the TXA cohort. There were no significant differences in demographics, surgical outcomes, blood loss, transfusion requirement, or perioperative hematologic and coagulation laboratory values between the two cohorts. Adverse events were similar between the groups, but did include two seizure events and two thromboembolic events related to vascular access devices.

DISCUSSION: We found no significant difference in blood loss, transfusion requirements, hematologic parameters, or outcomes between pediatric CCVR patients who received EACA vs. TXA. Further research is needed to define optimal antifibrinolytic dosing and duration of therapy. While standard laboratory parameters were similar between groups, future studies investigating coagulation-based and inflammatory assays may be useful in defining surgical-induced coagulopathy.

\section{Introduction}

Craniosynostosis, a condition of premature cranial suture fusion, occurs in approximately 1 in every 2,000 births. ${ }^{1}$ These patients often need complex surgical repair to correct skull deformities, and potentially prevent functional neurologic and developmental compromise. ${ }^{1,2}$ Complex cranial vault reconstruction (CCVR) is performed primarily in infants and young children and is an intricate and high-risk procedure, often requiring extensive scalp dissection and multiple skull osteotomies. ${ }^{1}$ Children undergoing surgical correction of craniosynostosis often experience high rates of bleeding and blood product transfusion, increasing the risk of postoperative complications including mortality. ${ }^{3-5}$ The most severe and most common perioperative issues relate to the rate and extent of blood loss, which can be up to several times the patient's total blood volume ${ }^{3,6,7}$ Contributing to high blood loss rates are the large surgical surfaces exposed, hyperfibrinolysis, 
and dilutional coagulopathy. ${ }^{6,8-10}$ This is a type of controlled traumatic coagulopathy for which the precise underlying mechanisms are not fully defined. ${ }^{3,6,8}$ Other reported complications include intraoperative cardiac arrest, venous air embolism, hypotension, bradycardia, postoperative seizures, surgical site infections, facial swelling with airway compromise, and unplanned postoperative mechanical ventilation. ${ }^{2,7,9,11-13}$ At our institution, approximately 30-40 children with craniosynostosis undergo CCVR each year. In the United States, 2000 children undergo CCVR per year, representing a unique opportunity to understand and improve rates of perioperative blood loss. ${ }^{14}$

One recently endorsed strategy for decreasing blood loss in craniosynostosis repair is the intraoperative use of antifibrinolytic agents. $\varepsilon$-Aminocaproic acid (EACA) and tranexamic acid (TXA) are synthetic lysine analogs that block the lysine binding sites on plasminogen, resulting in antifibrinolytic activity through inhibition of plasmin formation. ${ }^{10,15}$ Antifibrinolytics have been shown to reduce transfusion needs in surgical and trauma-related bleeding. ${ }^{10,16}$ TXA has been shown to be effective in limiting blood loss in infants and children undergoing CCVR, and two recent retrospective reviews suggest a benefit of EACA in this population as well. ${ }^{5,14,17-19}$ While the use of EACA and TXA to limit surgically-induced hemorrhage and transfusion requirements during CCVR has been extensively studied, the comparison of EACA vs TXA in children undergoing CCVR has not yet been evaluated. The aim of this study was to compare perioperative blood loss and need for transfusion in children receiving EACA and TXA. We hypothesized that TXA is associated with a greater decrease in blood loss and transfusion requirements when compared to EACA. A secondary aim was to identify possible laboratory predictors of blood loss in CCVR with the ultimate goal of trying to better understand surgical-induced coagulopathy in this population.

\section{Methods}

\section{Study Design and Setting}

This single-center retrospective observational cohort study was approved by the Vanderbilt University Medical Center Institutional Review Board (IRB\#191843) with a waiver of informed consent and HIPAA authorization. We identified pediatric patients (birth to 12 years) who had undergone CCVR at Monroe Carell Jr. Children's Hospital at Vanderbilt (MCJCHV) between September 1, 2015 and December 31, 2019. Using the electronic health record (EHR), we extracted information from the date of surgery to 30 days postoperatively. We collected clinical information including demographics, suture type, blood loss, transfusion data, hematologic laboratory parameters, antifibrinolytic medication use, ICU and total hospital length of stay, and adverse outcomes. We recorded the following clinical laboratory values during the perioperative period: hematocrit, platelet count, prothrombin time (PT), activated partial thromboplastin time (PTT), and fibrinogen. Blood loss measurements included intraoperative estimated blood loss, postoperative drain volumes, calculated blood loss, and estimated blood volume ${ }^{10}$ :

\section{Outcomes and Definitions}

Calculated blood loss and estimated blood volume were defined as follows ${ }^{10}$ :

$\mathrm{ERCV}_{\text {lost }}=\mathrm{ERCV}_{\text {preop }}+\mathrm{ERCV}_{\text {transfused }}-\mathrm{ERCV}_{\text {postop }}$

$\mathrm{EBV}_{\text {lost }}(\mathrm{mL} / \mathrm{kg})=\mathrm{ERCV}_{\text {lost }}(\mathrm{mL}) /\left[\mathrm{wt}(\mathrm{kg}) \times\right.$ Hct $\left._{\text {preop }} / 100\right]$

$\mathrm{EBV}=80 \mathrm{~mL} / \mathrm{kg}$ (for infants $<12$ months)

$\mathrm{EBV}=75 \mathrm{~mL} / \mathrm{kg}$ (for infants $>12$ months)

$[\mathrm{ERCV}=$ estimated red cell volume, $\mathrm{EBV}=$ estimated blood volume, Hct $=$ hematocrit, $\mathrm{RBC}=$ red blood cell]

\section{Data Collection and Statistical Analysis}

Data were collected and stored electronically, in accordance with HIPAA and in a de-identified format, using a registry identification number as an identifier. Data were analyzed using a statistical software package $(\mathrm{R}$ 3.6.3) with the assistance from the Department of Pediatrics and Biostatistics. Continuous variables were 
presented as median and interquartile range (IQR) and categorical variables were presented as frequency and percentage. EACA and TXA patients were compared using Wilcoxon rank sum test for continuous variables and Chi-square test for categorical variables. Ordinal logistic regression model was used to find the transfusion factors that associate with intraoperative and postoperative blood loss adjusting for age, weight, operative time, surgical vault category (anterior / fronto-orbital advancement, mid-posterior, or total), and syndromic status.

Results

\section{Cohort Demographics and Intraoperative Management}

We identified 95 patients (5 to 35 months of age) who underwent CCVR at MCJCHV between September 2015 and December 2019. All of the patients were cared for by the same pediatric plastic surgeon and pediatric neurosurgeon. Overall, 47 patients received EACA and 48 received TXA. Dosing regimen was largely equivalent for patients in each antifibrinolytic group, although a few patients received slightly different dosing regimen based on the anesthesiologist's preference. These minor dosing variations were equal between groups. There were no major differences in demographics between the two cohorts, except that there were slightly more syndromic patients in the EACA group (Table 1). There was no difference in operative time or ICU length of stay, but the total hospital length of stay was slightly longer in the TXA group. There were more intraoperative complications in the EACA group and more postoperative complications in the TXA group, but neither reached statistical significance (Table 1). Intraoperative complications included hypotension and possible venous air embolism. Postoperative complications included two patients with newonset seizure activity, one minor local allergic reaction, three patients requiring repeat operation (two for infectious complications, one for proptosis), one transfusion reaction, and two postoperative thromboembolic events (Table 2). Both postoperative thromboembolic events were associated with arterial lines placed for the procedure. One did not require any intervention. The other was treated with anticoagulation for $\sim 2.5$ months with complete resolution.

\section{Fluid Management and Transfusions}

There were no differences in intraoperative crystalloid or $5 \%$ albumin administration. Intraoperative blood product transfusion was also equal between groups. Overall, $62 \%$ of patients received intraoperative red blood cell (RBC) transfusion with an average volume of $15 \mathrm{~mL} / \mathrm{kg}$. Only one patient, who was in the EACA group, received intraoperative fresh frozen plasma (FFP) and platelet transfusions. Cryoprecipitate was given to $13 \%$ of all patients intraoperatively. There was a higher percentage of patients receiving cryoprecipitate in the EACA group, but this was not statistically significant. Postoperatively, $23 \%$ percent of patients received $\mathrm{RBC}$ transfusion, with an average volume of $3 \mathrm{~mL} / \mathrm{kg}$. One patient in each group received postoperative FFP, which was given for elevated INR without clinical symptoms. Only one patient received postoperative cryoprecipitate, which was given for hypofibrinogenemia without evidence of bleeding. No patients received postoperative platelet transfusion. Overall there was no difference in transfusion of blood products between groups (Table 3 ).

Blood Loss

There was no difference in estimated blood loss $(\mathrm{mL} / \mathrm{kg})$ and calculated blood loss $(\mathrm{mL} / \mathrm{kg})$ between the two groups (Table 4). Intraoperative calculated blood loss overall for all patients was $34 \mathrm{~mL} / \mathrm{kg}$, which equated to $44 \%$ blood volume loss. Postoperative calculated blood loss was $12 \mathrm{~mL} / \mathrm{kg}$ for the entire study cohort, amounting to $15 \%$ blood volume loss. There was slightly more drain output recorded in the EACA group during the 48 to 72 postoperative hours but no difference was observed for total drain output.

\section{Laboratory Parameters}

We collected hematologic and coagulation laboratory values on each patient in the preoperative, intraoperative, and postoperative setting. Laboratory values were collected up until the time of discharge. The minimum hematocrit, platelet count, and fibrinogen values were recorded, while the maximum PT and PTT were recorded. There were no differences in the minimum or maximum values of the collected laboratory 
parameters in the preoperative, intraoperative, immediate postoperative, or postoperative time periods (Table 5). Postoperative laboratory parameters are depicted in spaghetti plots in Supplementary Figure 1. Hematocrit dropped as expected in the intraoperative period and many patients experienced a second drop in the postoperative period, but nadir and recovery were highly variable. No patients developed significant thrombocytopenia during the perioperative period. There was a trend toward a small peak in PT around 24-36 hours postoperative, but this was also variable with several outliers. PTT tended to rise with time in the postoperative period, but this was only collected on a few patients $(n=11)$. Hypofibrinogenemia was not seen and fibrinogen activity tended to increase postoperatively.

\section{Laboratory Predictors of Blood Loss}

We evaluated several laboratory parameters to determine if they were could be predictive of blood loss (Table 6). Minimum intraoperative platelet count was predictive of both intraoperative and postoperative calculated blood loss. Minimum intraoperative fibrinogen and maximum PT were predictive of intraoperative blood loss, while maximum intraoperative PTT was predictive of postoperative blood loss. Postoperative laboratory values were not predictive of postoperative blood loss.

Discussion

Children undergoing surgical correction of craniosynostosis often experience high rates of bleeding and a type of controlled traumatic coagulopathy for which the mechanisms and optimal interventions are currently unknown. ${ }^{3,20,21}$ Antifibrinolytic therapy has been proven to be efficacious in reducing blood loss and need for transfusion in this population, but the relative efficacy of EACA vs. TXA is unknown. ${ }^{17,19,22,23}$ Likewise, the optimal dosing and duration of antifibrinolytic therapy remain unknown. ${ }^{14,23}$ The primary goal of this study was to compare the rates of blood loss and transfusion in children receiving EACA vs. TXA. At our institution, there was a transition in type of antifibrinolytic agent used from EACA to TXA at the end of 2017, which was primarily based on drug availability. Other factors, including surgeons and anesthesia protocols, remained equal across that time period. In our cohort, we did not find any significant difference in blood loss, transfusion requirements, hematologic parameters, or adverse outcomes between pediatric CCVR patients who received EACA and TXA.

Our cohort was similar to other large pediatric craniosynostosis cohorts in terms of age, weight, gender, type of procedure, and ASA status, though we did have fewer patients with syndromic craniosynostosis compared to many of the published groups. ${ }^{3,5,14,17,23}$ Intraoperative fluid management was in line with previously published studies. ${ }^{17,19,23}$ Transfusion rates in our cohort were lower than those previously reported in the literature, though similar to recent reports from single institutions. A large multicenter study $(n=1,814)$ that included 33 institutions reported transfusion of RBC-containing products in nearly $90 \%$ of patients, FFP in over $20 \%$ of patients and platelets and cryoprecipitate in $2-3 \%$ of patients. ${ }^{5}$ Average RBC transfusion volume was $30 \mathrm{~mL} / \mathrm{kg}$. In our cohort, only $62 \%$ of patients received RBC transfusion, and transfusion volume was lower at $15 \mathrm{~mL} / \mathrm{kg}$. Other groups, however, have shown a more significant reduction in transfusion rates, such as Dadure et al. who showed a reduction in total transfusion from $70 \%$ in a placebo group to $37 \%$ in a group given TXA. ${ }^{17}$ Goobie et al. likewise showed a reduction in blood loss, transfusion volume, and even avoidance of all postoperative blood product transfusion in a pediatric CCVR cohort receiving TXA compared to placebo. ${ }^{23}$

We collected and analyzed intraoperative estimated blood loss and postoperative drain output volumes as measures of blood loss in our cohort. However, we know these measurements are highly variable and unreliable as indicators of true blood loss. ${ }^{24}$ We found that calculated blood loss in our cohort was higher than estimated blood loss and that blood loss continued into the postoperative period. All patients in our cohort received antifibrinolytic therapy, and there was no observed difference in calculated blood loss or estimated blood loss between the EACA and TXA groups. Post-operative drain output was statistically higher in the EACA group, but likely of no clinical significance for the volumes reported. Furthermore, not all patients had drains placed during surgery.

We looked carefully at postoperative complications with respect to possible side effects from antifibrinolytic 
therapy. While TXA and EACA are relatively safe and inexpensive medications, there is concern about the potential for thrombosis, increasing risk of seizures, and potential interference with the fibrinolysis necessary for wound healing. ${ }^{25}$ There were two thromboembolic events, but both related to arterial access devices (not venous thromboembolism) and both relatively minor in severity. Two patients did develop seizure activity post-operatively, one with concern with baseline increased potential based on underlying diagnosis. The other without clear etiology, but self-limited. One patient did have significant surgical site wound infection, but wound healing was not otherwise an issue in this cohort. A large meta-analysis of adult surgical trials using TXA did not show any increased risk for stroke, deep vein thrombosis, pulmonary embolism, myocardial infarction, or death. The adult data do show, however, that TXA is efficacious in decreasing blood transfusion. ${ }^{10,26-28}$ Without any evidence of a difference in outcomes between pediatric craniofacial surgery patients receiving EACA vs TXA, centers may consider other factors such as cost, drug availability, and safety. ${ }^{29,30}$ Aminocaproic acid is more cost-effective in most settings, ${ }^{29,31,32}$ although there have been more issues with drug supply. There may be a slightly higher risk of seizures with TXA ${ }^{33}$, but there is not yet robust pediatric data to support this finding from retrospective review of adult surgical patients. Our center's current practice is to use tranexamic acid, based primarily on drug availability. However, as we accumulate more data about the use of antifibrinolytic agents in children undergoing surgery, it will be important to keep cost, efficacy, and safety considerations in mind.

In this study, we tried to identify laboratory parameters associated with blood loss and transfusion to obtain a better understanding of the mechanisms of surgical coagulopathy in this population. Intraoperative laboratory values did predict perioperative calculated blood loss. A lower intraoperative platelet count and fibrinogen predicted decrease intraoperative CBL. Lower intraoperative platelet count also predicted decreased CBL. However, it is important to note that none of these patients were thrombocytopenic nor hypofibrinogenemic. In contrast, a higher intraoperative prothrombin time was associated with increased intraoperative CBL and a higher postoperative partial thromboplastin time was associated with increased postoperative CBL, possibly reflecting procoagulant protein losses or a more abnormal coagulation state. While there were no differences in laboratory parameters between the two antifibrinolytic groups, there were some interesting postoperative laboratory trends that suggest ongoing disturbance of the hemostatic system beyond the operative period. Our data set was insufficient to draw firm conclusions about coagulopathy in this period but suggests that future prospective laboratory-based studies looking at coagulation assays may help elucidate some of the mechanisms of this surgical induced coagulopathy. In a retrospective study, we were limited to analysis of laboratory markers collected by the clinical team, but it would be interesting to consider additional markers of coagulation and fibrinolysis, including D-dimer, plasminogen activity, and plasmin-antiplasmin complexes. Better understanding the mechanisms of coagulopathy in craniofacial surgery and making optimal use of antifibrinolytic therapy has the potential to improve bleeding and surgical outcomes for children with craniosynostosis. A prospective trial comparing EACA and TXA and analyzing perioperative hematologic parameters would be beneficial in confirming the findings in our study.

\section{Conclusion}

In this single center study, no significant difference was found in blood loss, transfusion requirements, hematologic parameters, or outcomes between pediatric patients who received intraoperative EACA and TXA during CCVR for craniosynostosis correction.

\section{Conflict of Interest Statement}

The authors declare that the research was conducted in the absence of any commercial or financial relationships that could be construed as a potential conflict of interest.

\section{Acknowledgements}

The authors would like to thank the patients and families who make this work possible.

\section{References}


1. Cladis FP, Grunwaldt L, Losee J. Anesthesia for Pediatric Plastic Surgery. In: Smith's Anesthesia for Infants and Children. 9th ed. Elsevier; 2017:843-864.e3.

2. Faberowski L, Black S, Mickle J. Blood Loss and Transfusion Practice in the Perioperative Management of Craniosynostosis Repair. J Neurosurg Anesthesiol . 1999;11(3):167-172.

3. Stricker PA, Goobie SM, Cladis FP, et al. Perioperative Outcomes and Management in Pediatric Complex Cranial Vault ReconstructionA Multicenter Study from the Pediatric Craniofacial Collaborative Group.Anesthesiol J Am Soc Anesthesiol . 2017;126(2):276-287.

4. Goobie SM, Zurakowski D, Isaac KV, et al. Predictors of perioperative complications in paediatric cranial vault reconstruction surgery: a multicentre observational study from the Pediatric Craniofacial Collaborative Group. Br J Anaesth . 2019;122(2):215-223.

5. Fernandez PG, Taicher BM, Goobie SM, et al. Predictors of transfusion outcomes in pediatric complex cranial vault reconstruction: a multicentre observational study from the Pediatric Craniofacial Collaborative Group. Can J Anesth Can Anesth . 2019;66(5):512-526.

6. Goobie SM, Haas T. Bleeding management for pediatric craniotomies and craniofacial surgery. Pediatr Anesth . 2014;24(7):678-689.

7. Kearney RA, Rosales JK, Howes WJ. Craniosynostosis: an assessment of blood loss and transfusion practices. Can J Anaesth . 1989;36(4):473-477.

8. Goobie S, Soriano S, Zurakowski D, McGowan F, Rockoff M. Hemostatic Changes in Pediatric Neurosurgical Patients as Evaluated by Thrombelastograph@. Anesth Analg . 2001;93(4):887-892.

9. Williams GD, Ellenbogen RG, Gruss JS. Abnormal Coagulation during Pediatric Craniofacial Surgery. Pediatr Neurosurg . 2001;35(1):5-12.

10. Ker K, Edwards P, Perel P, Shakur H, Roberts I. Effect of tranexamic acid on surgical bleeding: systematic review and cumulative meta-analysis. BMJ . 2012;344:e3054.

11. Munro IRMB, Sabatier REMD. An Analysis of 12 Years of Craniomaxillofacial Surgery in Toronto. Plast Reconstr Surg . 1985;76(1):29-35.

12. Ririe D, Lantz P, Glazier S, Argenta L. Transfusion-Related Acute Lung Injury in an Infant During Craniofacial Surgery. Anesth Analg . 2005;101(4):1003-1006.

13. Buntain SG, Pabari M. Massive Transfusion and Hyperkalaemic Cardiac Arrest in Craniofacial Surgery in a Child. Anaesth Intensive Care . 1999;27(5):530-533.

14. Goobie SM, Cladis FP, Glover CD, et al. Safety of antifibrinolytics in cranial vault reconstructive surgery: a report from the pediatric craniofacial collaborative group. Pediatr Anesth . 2017;27(3):271-281.

15. Stafford-Smith M, Phillips-Bute B, Reddan DN, Black J, Newman MF. The Association of $\varepsilon-$ Aminocaproic Acid with Postoperative Decrease in Creatinine Clearance in 1502 Coronary Bypass Patients. Anesth Analg . 2000;91(5):1085-1090.

16. Kozek-Langenecker SA, Afshari A, Albaladejo P, et al. Management of severe perioperative bleeding: guidelines from the European Society of Anaesthesiology. Eur J Anaesthesiol . 2013;30(6):270-382.

17. Dadure C, Sauter M, Bringuier S, et al. Intraoperative tranexamic acid reduces blood transfusion in children undergoing craniosynostosis surgery: a randomized double-blind study. Anesthesiology . 2011;114(4):856-861.

18. Oppenheimer AJ, Ranganathan K, Levi B, et al. Minimizing Transfusions in Primary Cranial Vault Remodeling: The Role of Aminocaproic Acid. J Craniofac Surg . 2014;25(1):82-86. 
19. Hsu G, Taylor JA, Fiadjoe JE, et al. Aminocaproic acid administration is associated with reduced perioperative blood loss and transfusion in pediatric craniofacial surgery. Acta Anaesthesiol Scand . 2016;60(2):158165.

20. Williams KM, Higman MA, Chen AR, et al. Successful treatment of a child with late-onset T-cell post-transplant lymphoproliferative disorder/lymphoma. Pediatr Blood Cancer . 2008;50(3):667-670.

21. Stricker PA, Shaw TL, Desouza DG, et al. Blood loss, replacement, and associated morbidity in infants and children undergoing craniofacial surgery. Pediatr Anesth . 2010;20(2):150-159.

22. Faraoni D, Goobie SM. The efficacy of antifibrinolytic drugs in children undergoing noncardiac surgery: a systematic review of the literature. Anesth Analg . 2014;118(3):628-636.

23. Goobie SM, Meier PM, Pereira LM, et al. Efficacy of Tranexamic Acid in Pediatric Craniosynostosis SurgeryA Double-blind, Placebo-controlled Trial. Anesthesiol J Am Soc Anesthesiol . 2011;114(4):862-871.

24. Lopez MM, Lee J, Morrison K, Hoffman C, Souweidane M, Ascherman JA. Calculated Blood Loss and Transfusion Requirements in Primary Open Repair of Craniosynostosis. Plast Reconstr Surg Glob Open . $2019 ; 7(2)$.

25. Calapai G. Systematic Review of Tranexamic Acid Adverse Reactions.J Pharmacovigil . 2015;03(04).

26. Fergusson DA, Hébert PC, Mazer CD, et al. A comparison of aprotinin and lysine analogues in high-risk cardiac surgery. $N$ Engl $J$ Med . 2008;358(22):2319-2331.

27. Neilipovitz DT, Murto K, Hall L, Barrowman NJ, Splinter WM. A randomized trial of tranexamic acid to reduce blood transfusion for scoliosis surgery. Anesth Analg . 2001;93(1):82-87.

28. Goobie SM, Zurakowski D, Glotzbecker MP, et al. Tranexamic Acid Is Efficacious at Decreasing the Rate of Blood Loss in Adolescent Scoliosis Surgery: A Randomized Placebo-Controlled Trial. J Bone Jt Surg . 2018;100(23):2024-2032.

29. Riaz O, Aqil A, Asmar S, et al. Epsilon-aminocaproic acid versus tranexamic acid in total knee arthroplasty: a meta-analysis study.J Orthop Traumatol Off J Ital Soc Orthop Traumatol . 2019;20.

30. Bradley KE, Ryan SP, Penrose CT, et al. Tranexamic acid or epsilon-aminocaproic acid in total joint arthroplasty? A randomized controlled trial. Bone Jt J . 2019;101-B(9):1093-1099.

31. Churchill JL, Puca KE, Meyer E, Carleton M, Anderson MJ. Comparing $\varepsilon$-Aminocaproic Acid and Tranexamic Acid in Reducing Postoperative Transfusions in Total Knee Arthroplasty. J Knee Surg . 2017;30(5):460-466.

32. Falana O, Patel G. Efficacy and Safety of Tranexamic Acid Versus -Aminocaproic Acid in Cardiovascular Surgery. Ann Pharmacother . 2014;48(12):1563-1569.

33. Martin K, Breuer T, Gertler R, et al. Tranexamic acid versus e-aminocaproic acid: efficacy and safety in paediatric cardiac surgery.Eur J Cardiothorac Surg . 2011;39(6):892-897.

Legends

Supplemental Figure 1 Spaghetti plots of patient postoperative laboratory values vs time post-procedure. A) Hematocrit levels (\%) vs. time (minutes), B) Platelet count $\left(10^{3} / \mathrm{mcL}\right.$ ) vs. time (minutes), C) Prothrombin time (s) vs. time (minutes), D) Partial thromboplastin time (s) vs. time (minutes), and E) Fibrinogen $(\mathrm{mg} / \mathrm{dL})$ vs. time (minutes).

\section{Hosted file}

2_18_21 Table 1 Antifibrinolytics pediatric craniosynostosis repair.pdf available https://authorea.com/users/370842/articles/510348-\%CE\%B5-aminocaproic-acid-eaca-vs-

at 
tranexamic-acid-txa-in-children-undergoing-complex-cranial-vault-reconstruction-forrepair-of-craniosynostosis

\section{Hosted file}

2_18_21 Table 2 Antifibrinolytics pediatric craniosynostosis repair.pdf available https://authorea.com/users/370842/articles/510348-\%CE\%B5-aminocaproic-acid-eaca-vstranexamic-acid-txa-in-children-undergoing-complex-cranial-vault-reconstruction-forrepair-of-craniosynostosis

\section{Hosted file}

2_18_21 Table 3 Antifibrinolytics pediatric craniosynostosis repair.pdf available https://authorea.com/users/370842/articles/510348-\%CE\%B5-aminocaproic-acid-eaca-vstranexamic-acid-txa-in-children-undergoing-complex-cranial-vault-reconstruction-forrepair-of-craniosynostosis

\section{Hosted file}

2_18_21 Table 4 Antifibrinolytics pediatric craniosynostosis repair.pdf available https : //authorea.com/users/370842/articles/510348-\%CE\%B5-aminocaproic-acid-eaca-vstranexamic-acid-txa-in-children-undergoing-complex-cranial-vault-reconstruction-forrepair-of-craniosynostosis

\section{Hosted file}

2_18_21 Table 5 Antifibrinolytics pediatric craniosynostosis repair.pdf available https://authorea.com/users/370842/articles/510348-\%CE\%B5-aminocaproic-acid-eaca-vstranexamic-acid-txa-in-children-undergoing-complex-cranial-vault-reconstruction-forrepair-of-craniosynostosis

\section{Hosted file}

2_18_21 Table 6 Antifibrinolytics pediatric craniosynostosis repair.pdf available https : //authorea.com/users/370842/articles/510348-\%CE\%B5-aminocaproic-acid-eaca-vstranexamic-acid-txa-in-children-undergoing-complex-cranial-vault-reconstruction-forrepair-of-craniosynostosis 